\title{
A perspectiva dos supervisores sobre a integralidade nas práticas dos médicos cubanos do Programa Mais Médicos
}

\author{
The supervisors' perspective on comprehensiveness among the Cuban \\ doctors participating in the Mais Médicos Program (More Doctors) \\ Cassiano Mendes Franco ${ }^{\mathbf{1}}$ Patty Fidélis de Almeida ${ }^{\mathbf{2}}$, Ligia Giovanella ${ }^{\mathbf{3}}$
}

DOI: $10.1590 / 0103-1104201912001$

RESUMO A supervisão no Programa Mais Médicos (PMM) contempla ações clínico-pedagógicas, com o objetivo de qualificar o cuidado, e se articula a relevante estratégia para fixação de médicos na atenção básica. Este artigo analisa a perspectiva dos supervisores sobre os médicos cubanos do PMM no município do Rio de Janeiro quanto à integralidade das práticas, explorada em suas dimensões da abordagem biopsicossocial do cuidado com orientação comunitária e do elenco ampliado de ações de promoção, prevenção e assistência. Foram realizados análise temática dos relatórios mensais de supervisão, entre 2013 e 2015, e grupo focal com supervisores. Os resultados apontam que os médicos cubanos no PMM desenvolvem leque amplo de ações, com inserção comunitária, enfoque preventivo, planejamento de atividades e bom relacionamento interpessoal. Desafios que não se restringiam ao PMM, em muitos casos, foram relacionados à abordagem centrada na pessoa, à saúde mental e à insuficiente realização de alguns procedimentos.

PALAVRAS-CHAVE Atenção Primária à Saúde. Integralidade em saúde. Conhecimentos, atitudes e prática em saúde. Recursos humanos em saúde.

1 Universidade Federal de São Carlos (UFSCar),

Departamento de Medicina - São Carlos (SP), Brasil. casmenfran@gmail.com

2 Universidade Federal Fluminense (UFF), Instituto de Saúde Coletiva (ISC) Niterói (RJ), Brasil.

${ }^{3}$ Fundação Oswaldo Cruz (Fiocruz), Centro de Estudos Estratégicos (CEE)

- Rio de Janeiro (RJ), Brasil
ABSTRACT Supervision in the Mais Médicos Program (PMM) contemplates clinical-pedagogical actions, with the aim to qualify care, and articulates with the relevant strategy of doctors' fixation in primary care. This article analyzes the supervisors' perspective about Cuban doctors of the PMM in the city of Rio de Janeiro regarding the comprehensiveness of practices, explored in its dimensions of the biopsychosocial approach of care with community orientation and the expanded range of promotion, prevention and care actions. Thematic analysis of the monthly reports of supervision were carried out, between 2013 and 2015, and focus group with supervisors. Results show that Cuban doctors in PMM develop a wide range of actions, with community insertion, preventive approach, planning of activities and good interpersonal relationship. Challenges that were not restricted to the PMM, in many cases, were related to an approach focused on the person, on the mental health and in the insufficient performance of certain procedures.

KEYWORDS Primary Health Care. Comprehensiveness in health. Health knowledge, attitudes, practice. Health manpower. 


\section{Introdução}

A crise da força de trabalho em saúde é um desafio global que compromete o acesso universal à saúde $\mathbf{1}$. A concentração de profissionais em áreas urbanas e regiões mais ricas, além de promover iniquidades, resulta em ineficiência para os sistemas de saúde 2-4 $^{2}$. No Brasil, as desigualdades e a suboferta de trabalhadores são anteriores à implantação do Sistema Único de Saúde (SUS) 5 .

As iniciativas governamentais para enfrentar as deficiências no provimento de profissionais se desenrolaram por meio de sucessivos programas de caráter fragmentado, pontual e de alcance modesto, sem sustentação em longo prazo ${ }^{6}$. Diferencia-se, nesse aspecto, o Programa Mais Médicos (PMM), por sua abrangência, magnitude e celeridade ${ }^{6}$. Estrutura-se em três eixos: provimento emergencial de médicos, formação e infraestrutura das Unidades Básicas de Saúde (UBS) ${ }^{7}$.

O eixo provimento se destaca pela rápida capilarização no território nacional, com incorporação de mais de 18 mil médicos, com distribuição em 73\% dos municípios do Brasil. Houve peso predominante de médicos cubanos (cerca de 60\%, em 2016), recrutados por meio de cooperação com Cuba, diferenciando-se por possuírem maior experiência e formação específica na Atenção Primária à Saúde (APS) ${ }^{8}$.

Dentro desse eixo, a supervisão é prevista pela Lei $\mathrm{n}^{0} 12.871^{7}$. O supervisor integra o programa, definindo-se como "profissional médico responsável pela supervisão profissional contínua e permanente do médico"7(7), com responsabilidade sobre o acompanhamento e a fiscalização das atividades de ensino-serviço do médico participante, em conjunto com o gestor municipal7. Inclui visitas mensais aos médicos nas UBS e encontros para aperfeiçoamento do processo de trabalho. A remuneração é condicionada à entrega mensal de relatórios das visitas em um Web Portfólio: https://sistemas.unasus.gov.br/webportfolio.

Os supervisores são atores-chave no PMM, especialmente para a análise da integralidade das práticas dos profissionais. As visitas periódicas, com olhar técnico e pedagógico sobre a atuação dos médicos e sua inserção nas equipes, documentadas nos relatórios, compõem profícuo material de estudo e trazem perspectiva privilegiada sobre o tema. Alguns estudos 9,10 já apontaram a pertinência da supervisão acadêmica em discutir e induzir a práticas mais consoantes com os princípios e as necessidades do SUS, como a integralidade.

Este artigo analisa a perspectiva dos supervisores sobre a integralidade das práticas dos médicos cubanos no PMM. A integralidade é um conceito polissêmico, cabendo diversos sentidos, entre os quais, uma perspectiva focada sobre as práticas profissionais na saúde $^{\mathbf{8}, 11}$. Nesse aspecto, a integralidade nos cuidados primários, ou comprehensiveness, costuma ser traduzida como abrangência ou ampliação da carteira de serviços. No Brasil, o ideário da Reforma Sanitária expande tal concepção" ao incorporar cuidados completos, com vasta gama de ações e serviços conforme necessidades de diversas ordens ${ }^{\mathbf{1 2}}$.

Identifica-se como cuidado integral, além da realização de vastos serviços de atenção às necessidades em todo o processo saúde-adoecimento, aquele que considere as esferas biomédicas, psicológicas e sociais do sujeito, inserido em comunidade ${ }^{11-13}$. Desse modo, a integralidade das práticas é compreendida e analisada neste trabalho a partir de duas dimensões: abordagem biopsicossocial do cuidado, com orientação comunitária e elenco de ações de promoção, prevenção e assistência ${ }^{13}$.

\section{Metodologia}

Foi realizado estudo qualitativo, a partir de fontes primárias e secundárias, no município do Rio de Janeiro, na Área Programática 3.1 (AP 3.1), território que apresentava o maior número de equipes de saúde da família e UBS com médicos do PMM, contando com 36 profissionais (todos cubanos) e seis supervisoras, em setembro de $2015^{14}$. 
Para analisar a percepção dos supervisores sobre as práticas médicas, utilizou-se análise temática dos relatórios de supervisão do $W e b$ Portfólio (outubro de 2013 a setembro de 2015) e de grupo focal com as supervisoras do território selecionado, realizado em agosto de 2016.

\section{Registros de supervisão do Web Portfólio}

Após consulta e autorização do Ministério da Saúde, foram disponibilizados na íntegra os relatórios armazenados no Web Portfólio, consolidados em planilha Excel ${ }^{\circledR 15}$. São compostos por onze seções de preenchimento facultativo, sendo identificados e analisados os dados relacionados aos profissionais do PMM que atuavam na AP 3.1, totalizando 632 relatórios no período de outubro de 2013 a setembro de 2015. Selecionaram-se para análise, devido à pertinência com o tema da integralidade das práticas, as seções 'processo de trabalho' e 'atuação do médico'.

Em 'processo de trabalho', com campo de redação livre, eram registrados aspectos relevantes sobre: reunião de equipe; acolhimento dos usuários e organização da agenda; e trabalho no território. Na seção 'atuação do médico', o supervisor poderia atribuir nota de 0 a 10 para compreensão ampliada dos problemas de saúde e atuação com empatia e construção de vínculos; anamnese, exame físico e uso racional de recursos diagnósticos; assiduidade e pontualidade; e atividades comunitárias e inserção no processo de trabalho da equipe. Obteve-se a média das notas dessas avaliações e calculou-se a nota média por item para cada médico. Os registros de aspectos positivos e negativos foram submetidos à análise temática, com cálculos de frequência, incluindo quantidade e proporções percentuais. A análise temática explorou categorias emergidas do material empírico em confronto com uma matriz previamente elaborada de componentes e categorias identificadas às dimensões da integralidade.

Os relatórios de supervisão do $W e b$ Portfólio se referem a informações de 44 médicos participantes e 11 supervisores, lotados no território selecionado ao longo do período de análise (2013 a 2015). Todos os médicos eram cubanos, sendo $24 \mathrm{mu}$ lheres e 20 homens, com média de 17 anos de formados.

\section{Grupo focal}

Em julho de 2016, atuavam seis supervisoras, e quatro participaram do grupo focal (S1, S2, S3 e S4). Foi construído um roteiro para sua condução, que foi mediada por um dos autores, com auxílio de um assistente como observador/relator. Os temas explorados foram: abordagem centrada na pessoa, orientação comunitária, elenco de ações dos médicos e integração das ações com outros profissionais da equipe e da rede de atenção. $\mathrm{O}$ material gravado e transcrito foi submetido à análise temática de conteúdo ${ }^{16}$. Isto é, procedeu-se à leitura horizontal e exaustiva para construção de categorias empíricas, leitura transversal a partir da matriz de análise e esclarecimento da lógica interna do grupo de supervisores sobre o contexto de realização da prática médica, conforme a integralidade, no PMM.

As supervisoras eram médicas de família e comunidade, com experiência na Estratégia Saúde da Família (ESF). O tempo de experiência na supervisão do PMM variava de seis meses a dois anos, com seis a dez médicos sob a supervisão de cada uma. Todas tiveram passagem pela APS do município e três eram preceptoras em Residência de Medicina de Família e Comunidade.

De acordo com a força dos relatos no $\mathrm{Web}$ Portfólio e no grupo focal, foram atribuídas à presença e à observância integral da categoria nas práticas dos médicos do PMM +++; à presença parcial ++; e à insuficiência da categoria + .

Em cumprimento com os princípios éticos, o estudo atendeu à Resolução no 466/2012, sendo aprovado pelos pareceres $1.526 .773 \mathrm{e}$ 1.558.250. 


\section{A perspectiva da supervisão sobre a integralidade das práticas dos médicos cubanos do PMM}

\section{Abordagem biopsicossocial do cuida- do com orientação comunitária}

$\mathrm{Na}$ análise da abordagem biopsicossocial do cuidado com orientação comunitária, aspectos da dinâmica do espaço territorial foram explorados por meio das intervenções sobre os riscos sanitários, ambientais e sociais do território.
Os relatórios de supervisão, no tema 'trabalho no território', na seção 'processo de trabalho', destacaram a violência urbana, limitando ações comunitárias e visitas domiciliares (tabela 1). Ressalta-se visão mais coletiva sobre a saúde pelos profissionais do PMM - somando quase $30 \%$ dos campos sobre trabalho no território (tabela 1) - por meio da realização de grupos, campanhas, ações a partir da análise de indicadores de saúde e atenção a fatores sociais, com impacto positivo sobre a equipe e a população: "Tem realizado visitas domiciliares $e$ está atento aos riscos sociais durante a visita no território".

Tabela 1. Temas preenchidos na seção 'processo de trabalho', nos relatórios de supervisão prática do PMM AP 3.1 do município do Rio de Janeiro, de outubro de 2013 a setembro de 2015

\begin{tabular}{|c|c|c|}
\hline Tema: Trabalho no território & $\mathrm{n}$ & $\%$ \\
\hline Violência urbana & 75 & 60,5 \\
\hline Realiza grupos e campanhas & 23 & 18,5 \\
\hline Ações a partir de análise de indicadores & 14 & 11,3 \\
\hline Falta de profissionais ou outros recursos na UBS & 8 & 6,5 \\
\hline Falhas de registro em prontuário & 4 & 3,2 \\
\hline Total & 124 & 100,0 \\
\hline Tema: Acolhimento dos usuários e organização da agenda & $\mathbf{n}$ & $\%$ \\
\hline Desenvolve estratégias para acolher demanda espontânea & 32 & 28,1 \\
\hline Falta de profissionais e outros recursos na UBS & 28 & 24,6 \\
\hline Boa integração com a equipe & 23 & 20,2 \\
\hline Prejuízos da demanda espontânea sobre a programada & 17 & 14,9 \\
\hline Sobrecarga de atendimento & 15 & 13,2 \\
\hline Vulnerabilidade do território & 10 & 8,8 \\
\hline Dificuldades com usuários e membros da equipe & 7 & 6,1 \\
\hline Total & 114 & 100,0 \\
\hline Tema: Reunião de equipe & $\mathbf{n}$ & $\%$ \\
\hline Organiza os atendimentos & 34 & 37,4 \\
\hline Discute casos & 27 & 29,7 \\
\hline Boa interação com outros profissionais & 19 & 20,9 \\
\hline Ausência no turno da reunião de equipe & 13 & 14,3 \\
\hline Analisa dados do prontuário eletrônico & 6 & 6,6 \\
\hline Total & 91 & 100,0 \\
\hline
\end{tabular}

Fonte: Elaboração própria, a partir de Web Portfólio ${ }^{15}$ 
A boa inserção na comunidade também pôde ser verificada na avaliação dos supervisores sobre o item 'atividades comunitárias e inserção no processo de trabalho da equipe', vinculado à seção 'atuação do médico', em que foi atribuída nota média de 8,6 (tabela 2).

Tabela 2. Média da avaliação dos médicos por item da seção 'atuação do médico' nos relatórios de supervisão prática do PMM AP 3.1 do município do Rio de Janeiro, de outubro de 2013 a setembro de 2015

\begin{tabular}{|c|c|c|c|}
\hline Item & Média de avaliação & № médicos avaliados & $\%$ por item \\
\hline \multirow{4}{*}{$\begin{array}{l}\text { Compreensão ampliada dos problemas de saúde e } \\
\text { atuação com empatia e construção de vínculos } \\
\text { (nota média } 8,7 \text { ) }\end{array}$} & $0,0-6,9$ & 3 & 7,3 \\
\hline & $7,0-7,9$ & 2 & 4,8 \\
\hline & $8,0-8,9$ & 22 & 53,7 \\
\hline & $9,0-10,0$ & 14 & 34,2 \\
\hline \multirow{4}{*}{$\begin{array}{l}\text { Anamnese, exame físico e uso racional de recursos } \\
\text { diagnósticos } \\
\text { (nota média 8,4) }\end{array}$} & $0,0-6,9$ & 2 & 4,8 \\
\hline & $7,0-7,9$ & 10 & 24,4 \\
\hline & $8,0-8,9$ & 17 & 41,5 \\
\hline & $9,0-10,0$ & 12 & 29,3 \\
\hline \multirow{4}{*}{$\begin{array}{l}\text { Assiduidade e pontualidade } \\
\text { (nota média 9,3) }\end{array}$} & $0,0-6,9$ & 1 & 2,4 \\
\hline & $7,0-7,9$ & 1 & 2,4 \\
\hline & $8,0-8,9$ & 9 & 22,0 \\
\hline & $9,0-10,0$ & 30 & 73,2 \\
\hline \multirow{4}{*}{$\begin{array}{l}\text { Atividades comunitárias e inserção no processo de } \\
\text { trabalho da equipe } \\
\text { (nota média 8,6) }\end{array}$} & $0,0-6,9$ & 3 & 7,3 \\
\hline & $7,0-7,9$ & 2 & 4,8 \\
\hline & $8,0-8,9$ & 22 & 53,7 \\
\hline & $9,0-10,0$ & 14 & 34,2 \\
\hline
\end{tabular}

Fonte: Elaboração própria, a partir de Web Portfólio15

A sobrecarga dos médicos foi mencionada em $13 \%$ dos relatórios, no tema 'acolhimento dos usuários e organização da agenda' (tabela 1), prejudicando a dedicação a atividades comunitárias: "tem realizado grupos de promoção da saúde devido à superlotação da agenda". Também concorreram a falta de profissionais e a falta de condições da UBS:

Unidade, por ter poucas salas e em condições péssimas, não permite que as equipes funcionem normalmente.

Médica com dificuldade de gestão da agenda, pois até a data da visita era a única médica para assistir a três equipes.
No grupo focal, as supervisoras relataram prática de intervenções no território, todavia, limitada pela sobrecarga de atendimento e pela violência urbana:

O médico faz grupo na comunidade... A gente passou por uma boca de fumo... O paciente recebeu o médico em casa, abriu as portas da casa dele, para ele poder fazer um grupo dentro da comunidade. (S1).

Aspectos relacionados à abordagem centrada na pessoa ${ }^{17}$ foram considerados nas avaliações do Web Portfólio nos itens 'compreensão ampliada dos problemas de saúde e atuação com empatia e construção de vínculos' (nota 
média 8,7) e 'anamnese, exame físico e uso racional de recursos diagnósticos' (nota média 8,4) (tabela 2), com avaliação geral bastante positiva sobre elementos do método clínico centrado na pessoa.

Na mesma seção, sobressaíram aspectos positivos. Houve 151 apontamentos sobre o bom relacionamento interpessoal, superando impressão negativa de $26,9 \%$ dos relatórios (tabela 3 ). Foram elencadas características da relação com outros profissionais e usuários e da personalidade do médico: "Segura, articulada, atenciosa [...]"; "Ótima relação com o paciente, com escuta qualificada e facilidade de comunicação".

Tabela 3. Frequência de menções a aspectos negativos e positivos da atuação do médico nos relatórios de supervisão prática do PMM AP 3.1 do município do Rio de Janeiro, de outubro de 2013 a setembro de 2015

\begin{tabular}{|c|c|c|}
\hline Aspectos positivos & $\mathrm{n}$ & $\%$ \\
\hline Bom relacionamento interpessoal & 151 & 55,5 \\
\hline Competência e capacidade técnica & 101 & 37,1 \\
\hline Cooperação e disposição & 71 & 26,1 \\
\hline Disciplina e comprometimento & 68 & 25,0 \\
\hline Organização para o trabalho & 42 & 15,4 \\
\hline Capacidade de aprendizagem & 31 & 11,4 \\
\hline Experiência profissional & 18 & 6,6 \\
\hline Boa adaptação ao idioma & 13 & 4,8 \\
\hline Total & 272 & 100,0 \\
\hline Aspectos negativos & $\mathrm{n}$ & $\%$ \\
\hline Dificuldade de adaptação ao idioma & 63 & 33,9 \\
\hline Não foram observados aspectos negativos & 52 & 28,0 \\
\hline Problemas de relacionamento interpessoal & 50 & 26,9 \\
\hline Falta de habilidade em informática & 33 & 17.7 \\
\hline Registro inadequado no prontuário & 30 & 16,1 \\
\hline Falta de inserção no processo de trabalho em equipe & 26 & 14,0 \\
\hline Necessidade de atualização clínica & 24 & 12,9 \\
\hline Deficiência na realização de procedimentos & 24 & 12,9 \\
\hline Problemas na organização da agenda & 11 & 5,9 \\
\hline Problemas no acolhimento à demanda espontânea & 8 & 4,3 \\
\hline Falta de assiduidade ou pontualidade & 6 & 3,2 \\
\hline Sobrecarga de trabalho & 6 & 3,2 \\
\hline Modelo de atendimento inapropriado & 5 & 2,7 \\
\hline Prescrição inadequada & 2 & 1,1 \\
\hline Total & 186 & 100,0 \\
\hline
\end{tabular}

Fonte: Elaboração própria, a partir de Web Portfólio15. 
Dos registros sobre aspectos negativos da atuação do médico, 26,9\% tratam de problemas de relacionamento interpessoal (tabela 3):

\section{[...] muita veemência nas opiniões.}

\section{[...] apatia, desinteresse, [...].}

Por vezes, a médica tem oscilações de humor, não tendo boa relação com outros colegas; por vezes, até mesmo com os usuários.

A dificuldade de adaptação ao idioma, também pontuada no grupo focal, foi o aspecto negativo mais citado nos relatórios, mais presente nos primeiros, junto com outras questões de adaptação cultural. Em contraste, $4,8 \%$ dos relatórios indicaram bom domínio da língua portuguesa como aspecto positivo, e $28 \%$ inexistência de aspectos negativos (tabela 3 ).

Sobre a abordagem centrada na pessoa, o grupo focal manifestou impressões ambíguas. Apontou-se que os médicos demonstravam preocupação com o cuidado, traziam conhecimento amplo sobre os usuários atendidos, buscavam criar vínculo e entender a vivência que o paciente tem da doença. Por outro lado, percebiam falhas de comunicação:

[...] existem ferramentas de intervenção clínica em que é importante explorar a percepção da doença. É importante se comunicar bem, e, muitas vezes, eles têm dificuldade de comunicação nessas questões mais sutis, mais subjetivas. (S3).

As supervisoras mencionaram dificuldades de manejo do método centrado na pessoa em um tempo curto de consultas:

Como eles não vivenciam isso, não são treinados na abordagem centrada na pessoa pela própria formação. Não conseguem fazer abordagem centrada na pessoa nas consultas 'curtinhas'. (S4).

Ponderou-se que o curso de especialização em saúde da família contribuiu para trazer algumas bases conceituais.

Duas supervisoras destacaram pouca consideração da participação do usuário no plano de cuidados, ressaltando relação médico-paciente mais distante, hierárquica, com predomínio da autoridade do médico.

Para o exercício das ações intersetoriais, as supervisoras compreendiam existir um desafio que ultrapassava a capacidade de qualquer médico na APS no município:

Acho que a intersetorialidade é dificílima para o médico brasileiro, quanto mais para o cubano. [...] Gente, isso é um desafio gigante para a APS do Rio de Janeiro. (S4).

Quando ocorriam, eram articuladas, principalmente, pela gerência das UBS.

Para o componente 'valorização dos determinantes sociais', na seção 'processo de trabalho', nos registros sobre a reunião de equipe ( $n=91), 30 \%$ mostraram atenção a casos de maior vulnerabilidade, priorizando-os para agendamento de consultas e visitas domiciliares (tabela 1). Contudo, no grupo focal, predominou a impressão de que os médicos do PMM apresentavam fragilidades na definição de prioridades.

Indicativos da participação ativa das coletividades na construção de projetos de saúde foram identificados no grupo focal pelo estabelecimento de parcerias na comunidade, representadas pelos grupos e pelas relações estabelecidas com lideranças locais na área adscrita:

Os médicos que acompanho fazem grupos com associação de moradores, lideranças comunitárias, sim. (S3).

Minha impressão é que depende do território e de uma relação preestabelecida. Eu tenho uma clínica, que é na Maré, em que as médicas cubanas têm uma relação maravilhosa com a associação de moradores e com alguns líderes comunitários. (S4). 


\section{Elenco de ações de promoção, pre- venção e assistência}

Na dimensão da integralidade das práticas - elenco de ações de promoção, prevenção e assistência -, foram examinadas categorias relativas aos componentes: carteira ampla de serviços (com destaque para procedimentos invasivos e grupos terapêuticos), atenção à demanda espontânea e condições prioritárias, prestação de serviços em diversos cenários, integração com outros serviços de saúde, interdisciplinaridade, planejamento das intervenções e uso de protocolos assistenciais ${ }^{\mathbf{1 3}}$.

Quanto à carteira de serviços, constatou-se que os médicos fazem a maioria dos procedimentos selecionados. No Web Portfólio, no tema 'reunião de equipe', observam-se menções sobre organização do atendimento, incluindo a programação para inserção de Dispositivo Intrauterino (DIU) (tabela 1). Contudo, insuficiência na realização de procedimentos foi citada nos aspectos negativos em 12,9\% dos relatórios (tabela 3). Semelhantemente ao grupo focal, os registros referiram resistência ou falta de capacitação para realização de suturas e inserção de DIU. A exigência da gestão foi um dos fatores apontados como determinantes para a realização dos procedimentos, e a sobrecarga de atendimento um dificultador, embora a supervisão negasse falta de infraestrutura das UBS.

A participação em grupos, na categoria carteira de serviços, foi registrada recorrentemente. Representou 18,5\% dos 124 apontamentos sobre trabalho no território (tabela 1). Como no grupo focal, revelou-se estratégica para lidar com a forte pressão assistencial: "[Os médicos] têm realizado grupos para tentar diminuir a demanda livre".

A participação em grupos de usuários foi afirmada como frequente no grupo focal. Destaca-se a fala de uma supervisora, explicitando que essa era uma prática corrente e estratégica para viabilizar o acompanhamento das condições prioritárias, porém, com uma abordagem mais pragmática do que reflexiva:
"Todos os meus supervisionados fazem grupo. [...] Mas muito associado a ver exames. [...] para dar conta de ver todo mundo"(S1). Sublinhou-se que o pouco tempo para promoção de saúde para qualquer médico da APS do município induz a uma prática baseada na transmissão de informações.

No Web Portfólio, a atenção à demanda espontânea e programada foi manifesta por diversos registros na discussão sobre agenda de atendimentos. $\mathrm{O}$ alto volume de marcações sem agendamento prévio provocava tensões no processo de trabalho. O tema sobre acolhimento dos usuários e organização da agenda $(\mathrm{n}=114)$ apresentou apontamentos sobre estratégias de acolhimento à demanda espontânea (28,1\%), prejuízos da demanda espontânea sobre a programada (14,9\%) e sobrecarga de atendimento (13,2\%) (tabela 1): "A médica apresenta dificuldades para organização da agenda e equilíbrio entre demanda espontânea e programada, sentindo-se sobrecarregada".

Em dez relatórios, destacou-se a vulnerabilidade do território, que, como observado no grupo focal, contribuiu para forte pressão assistencial (tabela 1):

Pela alta vulnerabilidade, observa-se um excessivo número de demandas espontâneas, inclusive em espaços definidos para outras atividades, como grupos e reuniões.

O grupo focal apontou adaptações para realizar agenda mínima, acesso avançado, acolhimento por equipe ou por escala entre as equipes da UBS. A integração com a equipe facilitou essas estratégias. Conforme a supervisão, o acolhimento à demanda espontânea foi incorporado como atividade de todos os médicos. Foi mencionado que profissionais do PMM tendiam a adotar modelo de acesso avançado para ampliar a capacidade de atendimento no mesmo dia. Essa forma de agendamento foi uma contribuição trazida pela supervisão, que se tornou oportuna à realidade dos médicos: "Na verdade, a maioria dos médicos fazem acesso avançado, inclusive. Eles agendam só pré-natal. Eles gostam do modelo" (S3). 
O grupo focal informou que as práticas dos médicos cubanos incorporavam a atenção à demanda programada, atendendo sem restrição a qualquer ciclo de vida. Duas supervisoras relataram que, no começo das atividades, houve resistência no atendimento a certas condições relacionadas à saúde da mulher e da criança, superadas por determinação da gestão local.

O grupo focal pontuou limitações nos casos de saúde mental por insuficiente capacitação para manejo desses problemas, além de dificuldades do idioma:

Bom, não são todos, também não é uma coisa só do Mais Médicos. Mas, primeiro, porque parece que em Cuba essa parte de saúde mental não é do generalista. Acaba indo para especialista. [...] Aí entra também a barreira da língua. $O$ arsenal da palavra também é muito importante na abordagem da saúde mental. (S3).

A integração com outros serviços de saúde, segundo o grupo focal, enfrenta severas limitações devido a dificuldades no sistema municipal de saúde. "A pergunta é: quem consegue?" (S1) - sintetizou uma das supervisoras. A comunicação direta com a atenção especializada ocorria com os profissionais do Núcleo de Apoio à Saúde da Família (Nasf) ou em algumas das UBS mistas, que contavam com especialistas, ainda assim, com barreiras organizacionais para o diálogo. Essencialmente, a troca de informações se dava pelas guias de referência e contrarreferência, com dificuldades no retorno.

Mesmo com limitações no acesso à atenção especializada e hospitalar, os médicos apresentaram alguma capacidade de realizar coordenação do cuidado dos usuários, segundo grupo focal. A utilização do Sistema de Regulação de vagas ambulatoriais (Sisreg) se tornou ferramenta potente para os médicos no acompanhamento de casos encaminhados:

Eu acho que quando eles começam a dominar um pouco mais o Sisreg - um pouco não, bastante -, têm alguns que começam a entender bem mais como um instrumento de coordenação de cuidado. (S4).

A habilidade da coordenação do cuidado era aspecto da experiência no sistema de saúde cubano:

Agora, os médicos que eu supervisiono têm essa capacidade de coordenar, tinham esse exercício. Mas aqui ficam mais restritos a quê? A mandar uma solicitação de um parecer para um reumatologista, e que nada vai ser respondido daqui a seis meses. (S2).

No componente da interdisciplinaridade, foi investigada relação com a equipe de enfermagem, os Agentes Comunitários de Saúde (ACS) e o Nasf. Com poucas exceções, os médicos mostraram ter boa relação com todos os membros da equipe e com o Nasf, segundo a supervisão. Articulavam-se para cuidado em conjunto e discutiam condutas.

No Web Portfólio, a interação com diversos membros da UBS foi frequentemente relatada positivamente, em espaços de reunião de equipe, discussão de casos em conjunto e atividades de educação continuada entre ACS. Nos aspectos positivos da atuação do médico ( $\mathrm{n}=272$ ), 26,1\% relatos expressaram cooperação e disposição dos médicos, e $25 \%$ disciplina e comprometimento (tabela 3 ), demonstrando predomínio de boa relação com outros profissionais: "Participativo, opinativo, interessado, desenvolveu um vínculo de confiança com o Nasf".

Nos aspectos negativos, $14 \%$ dos registros indicavam falta de inserção no processo de trabalho da equipe (tabela 3). A ausência do médico nas reuniões de equipe ou sua fraca participação, bem como dificuldades de comunicação e conflitos foram relatados:

Médica tem apresentado dificuldades de relacionamento com a enfermeira, prejudicando o espaco da reunião de equipe. 
Médico refere que tem dificuldades para se posicionar na reunião de equipe. Não é ouvido por outros profissionais.

O grupo focal apontou forte interação interprofissional, sendo estratégica para que os médicos cubanos conseguissem se inserir melhor no contexto da ESF e na realidade local:

Eles sabem muito trabalhar em equipe. Eles trabalham com o Nasf. [...] Acho que, em geral, têm muito boa relação com as enfermeiras. Conseguem fazer cuidado conjunto, conseguem fazer interconsulta, conseguem discutir, até atender junto. (S4).

Eles [em Cuba] não tinham essa figura do enfermeiro dentro do consultório, atendendo assim. Eu acho que eles gostaram. Eu vejo todos se relacionando muito bem. (S3).

Sobre o planejamento das intervenções, no Web Portfólio, houve impressão bastante positiva da capacidade de organização e planejamento dos médicos do PMM, sinalizando, ao contrário do grupo focal, uma visão mais ampliada sobre o trabalho na APS. Ações a partir da análise de indicadores do prontuário eletrônico constaram em 20 relatórios da seção 'processo de trabalho' (tabela 1), enquanto 42 registros marcaram como aspecto positivo “organização para o trabalho" (tabela 3):

Organização. Desejo que as coisas funcionem bem e avancem.

Médica possui um ótimo entendimento dos indicadores de saúde e das ações necessárias para modificá-los.

Consegue organizar o processo de trabalho de sua equipe.

O grupo focal apontou que os médicos utilizavam indicadores de desempenho do prontuário eletrônico para organizar o trabalho da equipe. Embora os médicos não fossem beneficiados com gratificações pelo alcance das metas, procuravam seguir o direcionamento da gestão:

Isso eles fazem bem [...]. Eles sabem seguir programa. Cuidar de hipertenso, cuidar de diabético. O que eles me contam é que trabalham assim em Cuba. O hipertenso tem tantas consultas por ano e já é tudo 'agendadinho'. (S4).

Quanto ao seguimento adequado de protocolos assistenciais, no Web Portfólio, 266 (73\%) relatórios consideraram anamnese, exame físico e uso racional de recursos diagnósticos, dentro da avaliação sobre atuação do médico, com nota entre 9,0 e 10,0 (média 8,4) (tabela 2). Houve 101 relatórios destacando competência e capacidade técnica dos médicos nos aspectos positivos (tabela 3). Exaltaram-se o uso adequado de diretrizes de tratamento e o bom preparo profissional: "Prima pela qualidade. $E$ ́ muito preocupada com o seguimento dos pacientes, com atuar de acordo com os protocolos".

Mencionou-se, ainda, disposição para o aprendizado (11,4\%) (tabela 3$)$ :

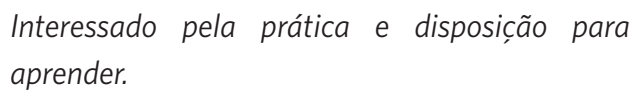

Disponibilidade para o aprendizado e para adaptar-se a novos protocolos clínicos.

Interesse acadêmico, sentimento de crescimento profissional [...].

Porém, atualização clínica foi sentida como uma necessidade em alguns relatórios (12,9\%). Ressalta-se que essa observação, contudo, foi minoritária em relação às menções positivas (tabela 3).

Sobre o uso de protocolos assistenciais, o grupo focal considerou que havia diferenças importantes entre os médicos, assim como a prática da Medicina Baseada em Evidências. Alguns apresentavam condutas pautadas em diretrizes atualizadas, porém, outros demonstravam práticas mais empíricas, com resistência 
a se orientar pelas recomendações das evidências científicas. Não obstante, destaca-se uma avaliação no grupo focal de que a vivência dos médicos no PMM contribuiu para fortalecer a sua resolutividade na APS e para desenvolver prática mais pautada pelas evidências científicas.

A síntese da avaliação de cada componente da integralidade nas práticas dos médicos cubanos do PMM, segundo supervisores, é apresentada no quadro 1.

Quadro 1. Avaliação da integralidade nas práticas dos médicos do PMM AP 3.1, do município do Rio de Janeiro, segundo supervisores, de 2013 a 2016

\begin{tabular}{|c|c|c|}
\hline \multicolumn{3}{|l|}{ Dimensão: abordagem biopsicossocial do cuidado, com orientação comunitária } \\
\hline Categorias de análise & Grupo focal & Web Portfólio \\
\hline Conhecimento da distribuição das condições de saúde & nsa & +++ \\
\hline Intervenção sobre os riscos do território & ++ & +++ \\
\hline Conhecimento e uso da abordagem centrada na pessoa & ++ & ++ \\
\hline Articulação com serviços de outros setores & + & nsa \\
\hline Atuação diferenciada a casos de vulnerabilidade social & + & +++ \\
\hline Formação de parcerias na comunidade & +++ & nsa \\
\hline \multicolumn{3}{|l|}{ Dimensão: elenco de ações de promoção, prevenção e assistência à saúde } \\
\hline Categorias de análise & Grupo focal & Web Portfólio \\
\hline Participação em grupos terapêuticos & +++ & +++ \\
\hline Realização de procedimentos invasivos de pequeno porte & ++ & ++ \\
\hline Atendimento à demanda espontânea & +++ & +++ \\
\hline Acompanhamento de condições prioritárias de saúde & ++ & +++ \\
\hline Atividades coletivas fora da UBS & nsa & +++ \\
\hline Visitas e/ou atendimentos domiciliares & +++ & ++ \\
\hline Troca de informações com profissionais da atenção secundária/terciária & + & nsa \\
\hline Acompanhamento dos casos encaminhados/coordenação do cuidado & ++ & nsa \\
\hline Reuniões de equipe, com formulação de plano de cuidado compartilhado & nsa & +++ \\
\hline Relação com enfermagem, ACS e Nasf & +++ & ++ \\
\hline Análise de indicadores de saúde da população & ++ & +++ \\
\hline $\begin{array}{l}\text { Conhecimento das linhas de cuidado definidas para a rede municipal e uso de } \\
\text { diretrizes nacionais e internacionais }\end{array}$ & ++ & +++ \\
\hline
\end{tabular}

A dimensão da integralidade, 'elenco de ações de promoção, prevenção e assistência', do ponto de vista dos supervisores, apresentou-se nas práticas dos médicos do PMM de forma mais potente que a dimensão 'abordagem biopsicossocial do cuidado, com orientação comunitária'. Constataram-se algumas divergências entre as impressões do grupo focal e dos relatórios do Web Portfólio. No grupo focal, a avaliação das supervisoras 
é mais crítica, destacando a insuficiência de algumas categorias (articulação com serviços de outros setores, atuação diferenciada em casos de vulnerabilidade social e troca de informações com profissionais da atenção secundária/terciária).

\section{Discussão}

Não obstante os questionamentos quanto ao potencial do PMM em ofertar atenção à saúde de qualidade à população $0^{6,8,9}$, os resultados deste estudo ratificam o alcance da integralidade das práticas, em graus distintos, conforme concepção adotada, com prestação de leque amplo de ações e serviços, coerente com a complexidade dos problemas de saúde e a pluralidade dos cenários. Os profissionais do PMM possuem marcada capacidade de inserção comunitária, enfoque preventivo, planejamento de ações e bom relacionamento interpessoal.

A forte integração territorial e a inserção comunitária condizem com achados de outros estudos sobre as práticas no $\mathrm{PMM}^{\mathbf{1 8 , 1 9}}$. A formação cubana, direcionada para a prestação de serviços voltados à comunidade ${ }^{20}$, ajuda a explicar a ocorrência desse achado. Identificamse acolhimento, vínculo, responsabilização e qualidade de atenção, indo ao encontro dos critérios para avaliação da APS sob a ótica da integralidade ${ }^{11}$. Estudos recentes corroboram tais resultados ${ }^{19,21}$.

Entre alguns pontos levantados pela supervisão, destacam-se coordenação da atenção, trabalho em equipe e acesso aberto às demandas dos usuários. A coordenação de cuidados, no caso do PMM, apesar da desarticulação entre níveis de atenção, apresentou-se como fator favorável às práticas integrais, diverso do observado em outros contextos da ESF22. A boa relação interpessoal e a integração interdisciplinar também obtiveram avaliação positiva, sendo importante para ampliação da oferta de ações e resposta mais efetiva às necessidades de saúde das pessoas ${ }^{23}$. Ressaltam-se achados semelhantes sobre acolhimento e interdisciplinaridade a outros estudos sobre práticas de médicos cubanos no $\mathrm{PMM}^{\mathbf{1 9}, 24}$.

As dificuldades apontadas não se restringem ao PMM em muitos casos. Limitações foram evidenciadas no sistema municipal de saúde, como pressão assistencial e violência, restringindo atividades comunitárias e intersetorialidade. Fragilidades externas à atuação dos médicos, interferindo na garantia das práticas integrais, são observadas em outros estudos ${ }^{8,25}$. Sobressai-se, nesta pesquisa, o forte tom crítico no grupo focal. Algumas falas enfatizam uma defesa da Medicina de Família e Comunidade brasileira, pouco permeável a experiências advindas de outras práticas e formações. Com efeito, o posicionamento inicial da Sociedade Brasileira de Medicina de Família e Comunidade ${ }^{26}$ argumentava pela preferência no investimento em formação na especialidade ao recrutamento de médicos estrangeiros.

Desafios foram sinalizados quanto à ampliação da autonomia das pessoas nas decisões clínicas, ao manejo mais potente de problemas de ordem psíquica, à sistematização de ferramentas de abordagem (centrada na pessoa e a casos vulneráveis, por exemplo) e à capacitação para procedimentos invasivos.

A abordagem centrada na pessoa foi verificada nos relatórios e no grupo focal. Porém, segundo avaliação do grupo, haveria baixa sistematização da técnica. A autonomia dos usuários em decisões sobre conduta foi um dos pontos controversos. $\mathrm{O}$ vínculo profissional-usuário exige que o paciente seja assumido como sujeito que fala, deseja e julga"1. Nesse sentido, Kolling ${ }^{27}$ aponta que negociar medidas apropriadas de conduta tem sido negligenciado na prática clínica.

O desafio de lidar com problemas de ordem psíquica ocorre no contexto geral da APS, em que se verifica que médicos costumam apresentar resistências em abordar casos de saúde mental ${ }^{28}$. Lima $^{29}$ ressalta que persistem constrangimentos em se conceber a comunidade como espaço de cuidado dos usuários de saúde mental.

Em contraste ao estudo de Girardi et al. ${ }^{\mathbf{8}}$, em que mais de $85 \%$ dos médicos relataram 
saber fazer procedimentos técnico-cirúrgicos selecionados, o grupo focal apontou que essa prática não era realizada por parte dos médicos, por falta de capacitação. Nesse sentido, vale ressaltar a potencialidade dos processos de aprendizagem relacionados à supervisão para melhoria e superação desses obstáculos. Engstrom et al. ${ }^{10}$ realçam essa potência de desenvolvimento da força de trabalho no SUS pela identificação de necessidades educacionais e ofertas de formação no ambiente do trabalho a partir da supervisão.

Observaram-se achados semelhantes aos de Thumé et al. ${ }^{30}$, que mostraram que médicos com larga experiência na APS e especialistas nessa área podem agregar novos saberes. $\mathrm{O}$ estudo mostrou que a supervisão apresentou conceitos, discutiu casos e condutas e realizou capacitações técnicas. Achados semelhantes foram discutidos por Almeida et al.9. Melo ${ }^{31}$ aponta que a supervisão tem potencialidades inexploradas e que apresenta limites ao ter foco no médico e não na equipe.

\section{Considerações finais}

Por fim, os resultados do estudo sinalizam que o Programa Mais Médicos tem ido além do acesso a consultas, procedimentos e da diminuição das iniquidades na distribuição de médicos. Destaca-se que a prática dos médicos cubanos possibilitou oferta de cuidados integrais em saúde, contribuindo para concepção abrangente da APS e fortalecimento do SUS. A contribuição dos médicos cubanos no acesso à assistência integral pelo SUS em áreas remotas e desfavorecidas no Brasil já se tornou histórica. Este artigo se soma a um conjunto de evidências que ratificam a importância da participação desses profissionais. Apesar de polêmicas muitas vezes infundadas sobre sua atuação, que foram maioria no PMM até a saída recente de Cuba da cooperação internacional com o Brasil, constata-se, nesta pesquisa, realizada no município do Rio de Janeiro, a integralidade em suas práticas.

\section{Colaboradores}

Franco CM (0000-0003-1430-6951)* participou substancialmente da concepção, planejamento, análise e interpretação dos dados e participou significativamente na elaboração do rascunho e da revisão crítica do conteúdo. Almeida PF (0000-0003-1676-3574)* participou substancialmente da concepção, planejamento, análise e interpretação dos dados, participou significativamente na revisão crítica do conteúdo e participou da aprovação da versão final do manuscrito. Giovanella $L$ (0000-0002-6522-545X)* participou substancialmente da concepção, planejamento, análise e interpretação dos dados, participou significativamente na revisão crítica do conteúdo e participou da aprovação da versão final do manuscrito. 


\section{Referências}

1. Campbell J. The route to effective coverage is through the health worker: there are no shortcuts. The Lancet. $2013 ; 381(9868): 725$.

2. Araújo E, Maeda A. How to recruit and retain health workers in rural and remote areas in developing countries: a guidance note. Washington: World Bank; 2013.

3. Portela GZ, Fehn AC, Ungerer RLS, et al. Recursos humanos em saúde: crise global e cooperação internacional. Ciênc. Saúde Colet. 2017; 22(7):2237-2246.

4. Liu X, Dou L, Zhang H, et al. Analysis of context factors in compulsory and incentive strategies for improving attraction and retention of health workers in rural and remote areas: a systematic review. Hum Res Health. 2015; 13(61):1.

5. Carvalho MS, Santos NR, Campos GWS. A construção do SUS e o planejamento da força de trabalho em saúde no Brasil: breve trajetória histórica. Saúde debate. 2013; 37(98):372-387.

6. Oliveira APC, Gabriel M, Poz MRD, et al. Desafios para assegurar a disponibilidade e acessibilidade à assistência médica no Sistema Único de Saúde. Ciênc. Saúde Colet. 2017; 22(4):1165-1180.

7. Brasil. Lei ${ }^{\circ} 12.871$, de 22 de outubro de 2013. Institui o Programa Mais Médicos, altera a Lei $n^{\circ} 8.745$, de 9 de dezembro de 1993, e $\mathrm{n}^{\circ}$ 6.932, de 7 de julho de 1981, e dá outras providências. Diário Oficial da União, 22 Out 2013.

8. Girardi SN, Carvalho CL, Pierantoni CR, et al. Avaliação do escopo de prática de médicos participantes do Programa Mais Médicos e fatores associados. Ciênc. Saúde Colet. 2016; 21(9):2737-2746.

9. Almeida ER, Martins AF, Macedo HM, et al. More Doctors in Brazil Project: an analysis of Academic Supervision. Interface (Botucatu). 2017; 21(supl1):12911300.
10. Engstrom EM, Carvalho LC, Romano VF, et al. O supervisor e as estratégias educacionais dos encontros locorregionais no Programa Mais Médicos do Brasil: reflexões acerca de concepções e práticas. Tempus (Brasília, DF). 2016; 10(1):241-252.

11. Silva Junior AG, Mascarenhas MTM. Avaliação da Atenção Básica em Saúde sob a Ótica da Integralidade: Aspectos Conceituais e Metodológicos. In: Pinheiro R, Mattos RA, organizadores. Cuidado: As fronteiras da Integralidade. Rio de Janeiro: Abrasco, São Paulo: Hucitec; 2004. p. 241-257.

12. Conill EM. Avaliação da integralidade: conferindo sentido para os pactos na programação de metas dos sistemas municipais de saúde. Cad. Saúde Pública. 2004; 20(5):1417-1423.

13. Franco CM, Almeida PF, Giovanella L. A integralidade das práticas dos médicos cubanos no Programa Mais Médicos na cidade do Rio de Janeiro, Brasil. Cad. Saúde Pública. 2018; 34(9):e00102917.

14. Cadastro Nacional dos Estabelecimentos de Saúde. Data-SUS: Informações de Saúde [internet]. 2015 [acesso em 2015 out 20]. Disponível em: http://cnes. datasus.gov.br/.

15. Brasil. Ministério da Saúde. Secretaria de Gestão e Educação do Trabalho em Saúde, Departamento de Planejamento e Regulação da Provisão de Profissionais da Saúde. Relatórios de Supervisão Prática (planilha Excel ${ }^{\varpi}$. Brasília, DF: Ministério da Saúde; 2015.

16. Minayo MCS. O Desafio do Conhecimento: Pesquisa Qualitativa em Saúde. 14. ed. São Paulo: Hucitec; 2014.

17. Stewart M, Brown JB, Weston WW, et al. Medicina centrada na pessoa: transformando o método clínico. 3. ed. Porto Alegre: Artmed. 2017.

18. Pereira LL, Silva HP, Santos LMP. Projeto Mais Médicos para o Brasil: Estudo de caso em Comunidades Quilombolas. Rev. ABPN. 2015; 7(16):28-51. 
19. Santos JBF, Maciel RHMO, Lessa MGG, et al. Médicos estrangeiros no Brasil: a arte do saber olhar, escutar e tocar. Saúde Soc. 2016; 25(4):1003-1016.

20. Keck CW, Reed GA. The curious case of Cuba. Am. j. public health. 2012; 102(8):13-20.

21. Liz RG, Lima RCGS. Users' perceptions on social impact of the cooperation project of the Mais Médicos Program: a case study. Interface (Botucatu). 2017; 21(supl1):1281-90.

22. Almeida PF, Santos AM, Souza MKB, organizadores. Atenção Primária à Saúde na coordenação do cuidado em Regiões de Saúde. Salvador: EDUFBA, 2015.

23. Ayres JRA. O cuidado, os modos de ser (do) humano e as práticas de saúde. Saúde e Soc. 2004; 13(3):16-29.

24. Organização Mundial de Saúde, Organização Panamericana de Saúde. Programa Mais Médicos no município do Rio de Janeiro: mais acesso, equidade e resolutividade na APS. Brasília, DF: OPAS, 2016. Série Estudos de Caso Sobre o Programa Mais Médicos - 2.

25. Pereira LL, Pacheco L. The challenges faced by the More Doctors Program in providing and ensuring comprehensive health care in rural areas in the Amazon region, Brazil. Interface (Botucatu). 2017; 21(supl1):1181-1192.

26. Sociedade Brasileira de Medicina de Família e Comunidade. Posicionamento em relação ao programa
“Mais Médicos” [internet]. 2013 [acesso em 2018 abr 23]. Disponível em: http://www.sbmfc.org.br/default. asp?site $\_$Acao $=$mostraPagina $\&$ paginaId $=909$.

27. Kolling MG. Método Clínico Centrado na Pessoa. In: Duncan BB, Schmidt MI, Giugliani ERJ, et al. Medicina ambulatorial: condutas de atenção primária baseadas em evidências. 4. ed. Porto Alegre: Artmed, 2013. 71-78.

28. Dourado GOL, Costa MCM, Soares ADAS, et al. Redução de danos no âmbito da estratégia saúde da família: estratégia reflexiva. Revista Interdisciplinar. 2014; 7(2):173-183.

29. Lima ED. Imaginário social sobre a loucura: cultura e práticas de cuidado em saúde mental. Curitiba: Appris; 2016.

30. Thumé E, Wachs LS, Soares MU, et al. Reflexões dos médicos sobre o processo pessoal de aprendizagem e os significados da especialização à distância em saúde da família. Ciênc. Saúde Colet. 2016; 21(9):28072814.

31. Melo EA. O que pode o Mais Médicos? Ciên. Saúde Colet. 2016; 21(9):2672-2674.

\footnotetext{
Recebido em 06/09/2018 Aprovado em 04/12/2018 Conflito de interesses: inexistente
}

Suporte financeiro: não houve 